\title{
ON DYNAMIC SOLUTION IN GEOMECHANICS USING THE FLAC-PROGRAM
}

\author{
Felix Kirzhner \\ Department of Geotechnics, Faculty of Civil Engineering \\ Technion, Haifa, Israel
}

\begin{abstract}
SUMMARY
An estimation of the accuracy of numerical dynamic solutions using the FLAG model was performed on a two-dimensional explicit finite-difference code. The paper had two complementary aims: (a) to indicate the feasibility of presentation of the velocity and acceleration by means of a damper, (b) to apply in the FLAC model a function of a longitudinal harmonic wave which acts on the medium. Data showed that fairly close agreement between analytical and numerical models was obtained.
\end{abstract}

\section{INTRODUCTION}

An approach to numerical dynamic analysis of soil-structure interaction was suggested by Cundall 11/. In the FLAC program $/ 2 /$, which is a code based on a two-dimensional explicit finite-difference scheme for dynamic modeling, three aspects were indicated as follows:

- external loading and boundary conditions;

- mechanical damping,

- wave transmission through the model.

The FLAC program has two main schemes: (a) Quiet boundaries (non-refracting) and (b) Free-Field boundaries. In the first scheme the modeling of problems of geomechanics involves media which, in the range of the analysis, are better represented as unbounded. Deep underground excavations are normally assumed to he within a half-space. In the second scheme, the seismic analysis of surface structures (such as dams) requires discretization of the region of the material adjacent to the foundation. The seismic input is normally represented by plane waves propagating upwards through the underlying material. The boundary conditions along the sides of the domain must allow for the free-field motion which would take place in absence of the structure.

FLAC uses a dynamic algorithm for solution of two general classes of mechanical problems: 
quasi-static and dynamic. In the dynamic analysis, damping in the numerical simulation is aimed at reproducing the energy losses of the natural system when subjected to a dynamic loading.

The present paper estimates the accuracy of the proposed numerical dynamic solution, using an example for illustration.

\section{BASIC EQUATION}

Consider the action of a longitudinal harmonic wave, propagating upwards (Fig. 1) in an elastic isotropic medium.

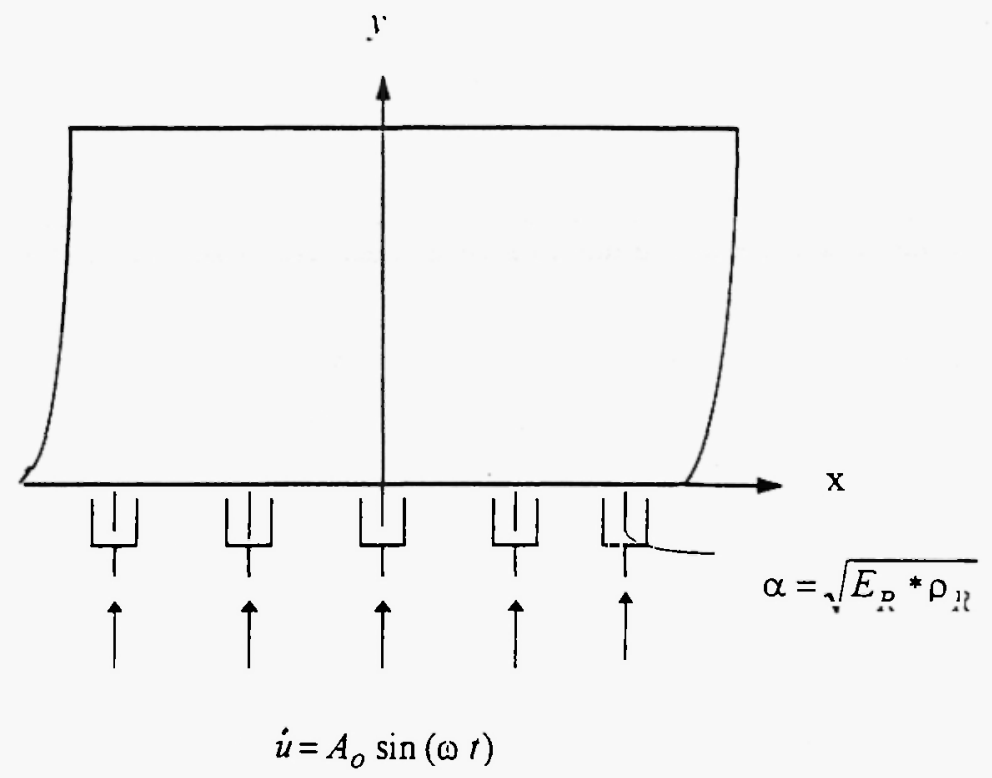

Fig. 1: The domain of the problem.

The FLAC program takes reflections into account by assuming that the motion is transmitted through a damper having the properties of the underlying material.

Let the velocity of the lower damper boundary be the following harmonic function of time:

$V_{1)}=A_{v} e^{i(1) l}$

where $\omega$ is the radian circular frequency of oscillation, and $t$ is time. The circular frequency of oscillation is:

$$
f=\frac{\omega}{2 \pi}
$$

The equation of the longitudinal oscillation, $u_{y}$ of the examined medium reads: 
$\rho \ddot{i}_{\nu}=\widetilde{E} \frac{\partial^{2} u_{\nu}}{\partial y^{2}}$

where $\rho$ is the density of the medium, and $\widetilde{E}$ is the modulus of longitudinal deformation in the absence of transverse displacement.

The modulus of the longitudinal deformation is

$$
\tilde{E}=E \frac{(1-v)}{(1+v)(1-2 v)}
$$

where $v$ is Poisson's ratio, and $E$ is Young's modulus.

In the steady state the displacements are

$u_{y}=u_{y 0} e^{i \omega t}$

and substitution into equation (2) yields:

$-\rho \omega^{2} u_{v .0}=\tilde{E} \frac{\partial^{2} u_{v .0}}{\partial y^{2}}$

or

$\frac{\partial^{2} u_{y, 0}}{\partial y^{2}}+\frac{\rho \omega^{2}}{\widetilde{E}} u_{y 0}=0$

The stress amplitude at $y=0$ is

$\left.\sigma\right|_{y=0}=\mu\left[\dot{u}_{y(0)}-V_{0}\right]$

with the damping factor:

$\mu=\sqrt{\widetilde{E} \rho}$

Denoting in Eq. (5) $\quad \lambda^{2}=\frac{\rho \omega^{2}}{\widetilde{E}}$. or $\lambda=\sqrt{\frac{\rho \omega^{2}}{\tilde{E}}}$

We write the suggested solution as:

$u_{\nu .0}=A \cos \lambda y+B \sin \lambda \nu$

Since there are no stresses at the upper boundary, we have

$\frac{\partial u_{y .0}(0)}{\partial y}=0$ 
whence

$B=A \operatorname{tg} \lambda H$

We use the condition (6) for determination of the coefficient A, as follows:

$\mu\left[i \omega u_{y .0}(0)-A_{v}\right]=\tilde{E} \frac{\hat{c} u_{y .0}(0)}{\partial y}$

and in view of (10), we have

$\mu\left[i \omega A-A_{\nu}\right]=\tilde{E} A_{v} \operatorname{tg} \lambda H$

whence

$A=\frac{\mu A_{v}}{\mu i \omega-\widetilde{E} \lambda \operatorname{tg} \lambda H}$

Since the solution of (8) has the form

$u_{v .0}=A[\cos \lambda y+\operatorname{tg}(\lambda H) \sin \lambda y]$

Then at $y=0$ the displacements $u_{y, 0}$ become

$$
\begin{aligned}
& u_{y}=A e^{i(s) t}=\frac{\mu A_{l}}{\mu i(j)-\tilde{E} \lambda \operatorname{tg}(\lambda H)} e^{i(1) t} \\
& u_{y}=\frac{\mu A_{1}[\tilde{E} \lambda \lg (\lambda H)+i \mu \omega]}{\mu^{2} \omega^{2}+\tilde{E}^{2} \lambda^{2} \operatorname{tg}^{2}(\lambda H)} i^{\prime(1) t}
\end{aligned}
$$

or

$u_{y}=-b_{0} e^{i\left(\omega t+\varepsilon_{0}\right)}$

with

$$
\begin{aligned}
& b_{n}=\frac{\mu A_{v}}{\sqrt{\mu^{2} \omega^{2}+\tilde{E}^{2} \lambda^{2} \operatorname{tg}^{2}(\lambda H)}} \\
& \operatorname{tg} \varepsilon=\frac{\mu \omega}{\widetilde{E} \lambda \operatorname{tg}(\lambda H)}
\end{aligned}
$$


Using an operative velocity function of the form

$V_{0}=A_{v} \sin \omega t$

yields after separation of the imaginary part, the displacement:

$u_{y}(0)=-b_{0} \sin (\omega t+\varepsilon)$

With the explicit expression for the argument $\varepsilon$ of the complex number:

$$
\varepsilon=\operatorname{arctg} \frac{\mu \omega}{\widetilde{E} \lambda \operatorname{tg}(\lambda H)}
$$

This way the displacement $u_{y}(0)$ is determined taking into account the oscillation amplitude $-b_{0}$

$$
b_{0}=\frac{\mu A_{v}}{a_{0}}
$$

\section{ILLUSTRATION}

For illustration, the following data is used:

$\begin{array}{lll}\text { Density } & - & \rho=2.18 \mathrm{~T} / \mathrm{m}^{3}, \\ \text { Elastic modulus } & - & \mathrm{E}=116 \mathrm{GPa}, \\ \text { Circular frequency } & - & \mathrm{f}=0.5 ; 1.0 ; 2.0 ; 4.0, \\ \text { Poisson's ratio } & - & v=0.4, \\ \text { Wave velocity } & - & A_{v}=1 \mathrm{~m} / \mathrm{sec}, \\ \text { Thickness of medium } & - & \mathrm{H}=100 \mathrm{~m} .\end{array}$

\section{RESULTS}

The numerica' solution is realized in the present paper using a very simple model and FLAC. A longitudinal harmonic wave is imposed on the medium. The computational scheme and boundary conditions are given in Fig. 2, and the applied velocity function, velocity function on the bottom of grid and the stress at $y=0$ are illustrated in Figures 3,4 and 5, respectively.

Table 1 compares the results of the analytical and the numerical models for different oscillation amplitudes. 


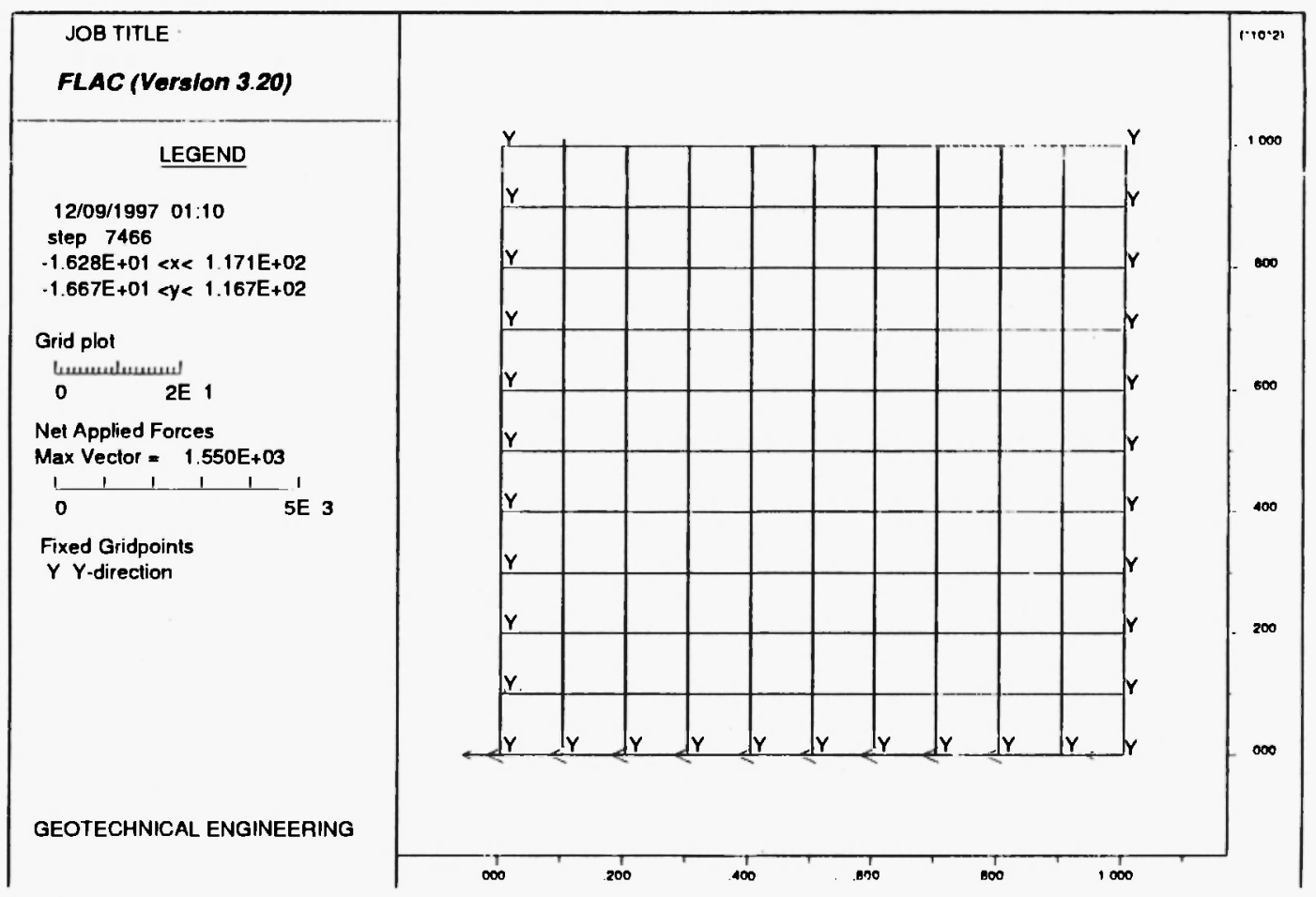

Fig. 2: The computation scheme and the boundary conditions of the numerical model.

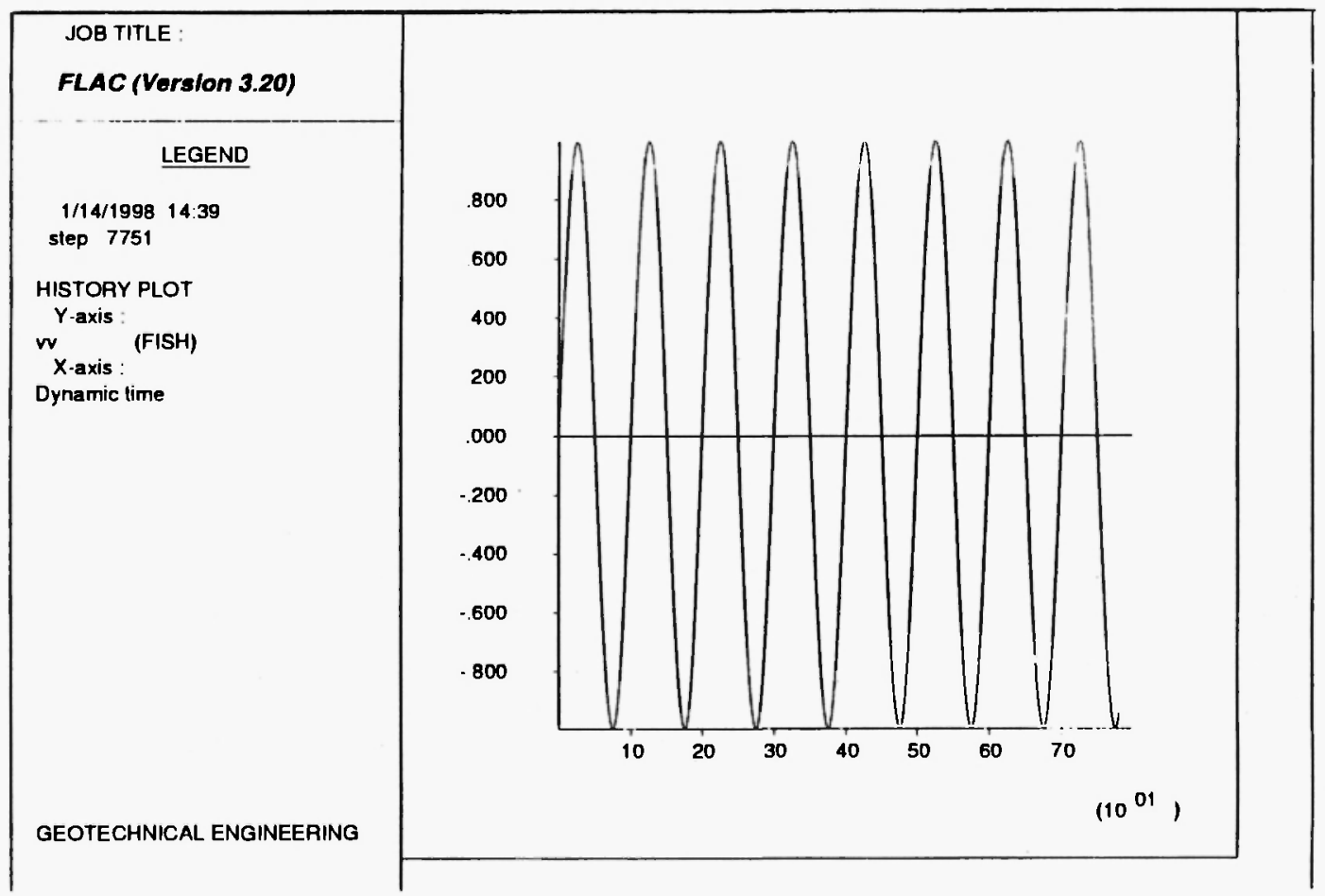

Fig. 3: The applied velocity function. 


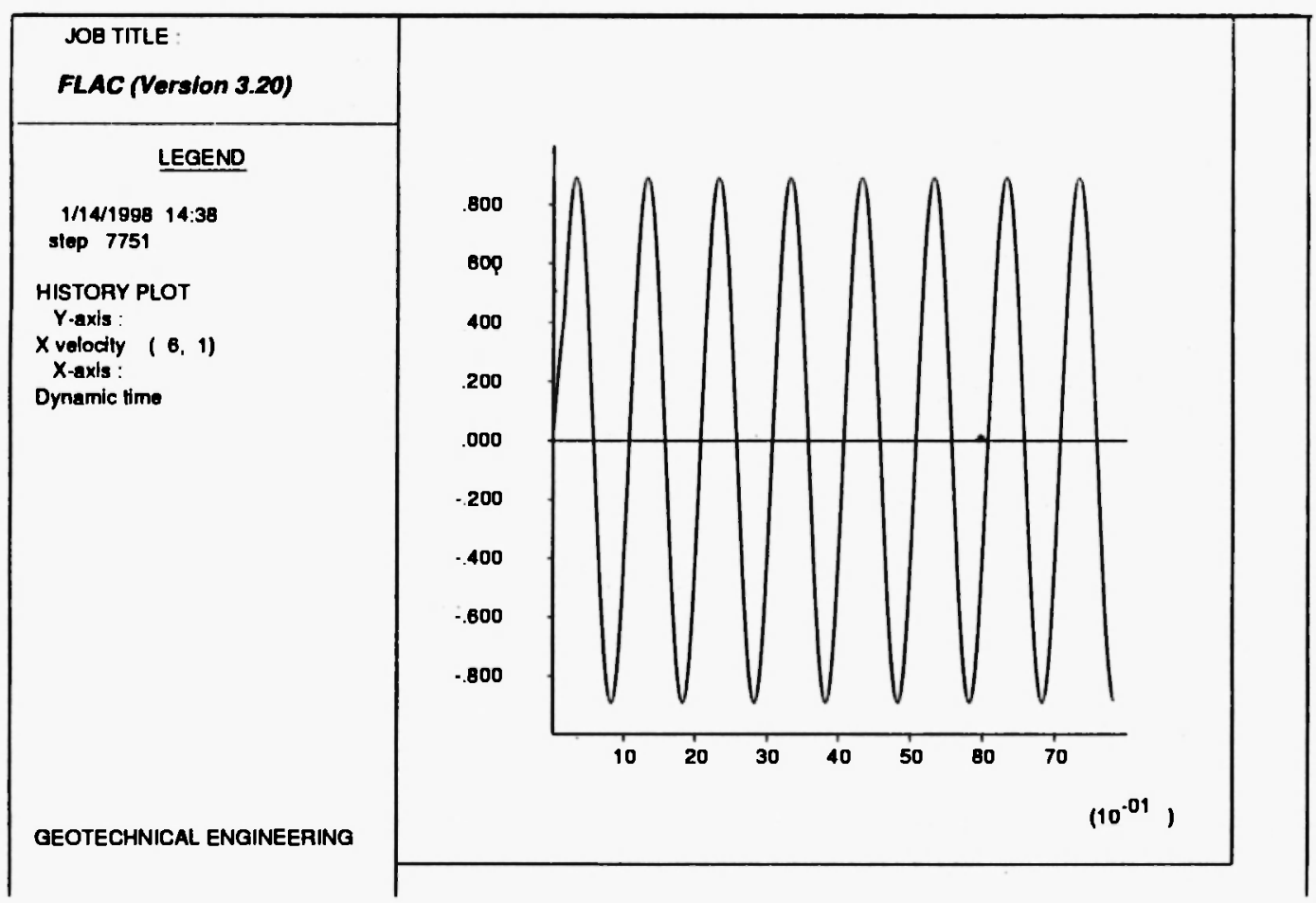

Fig. 4: The velocity function on the bottom of grid.

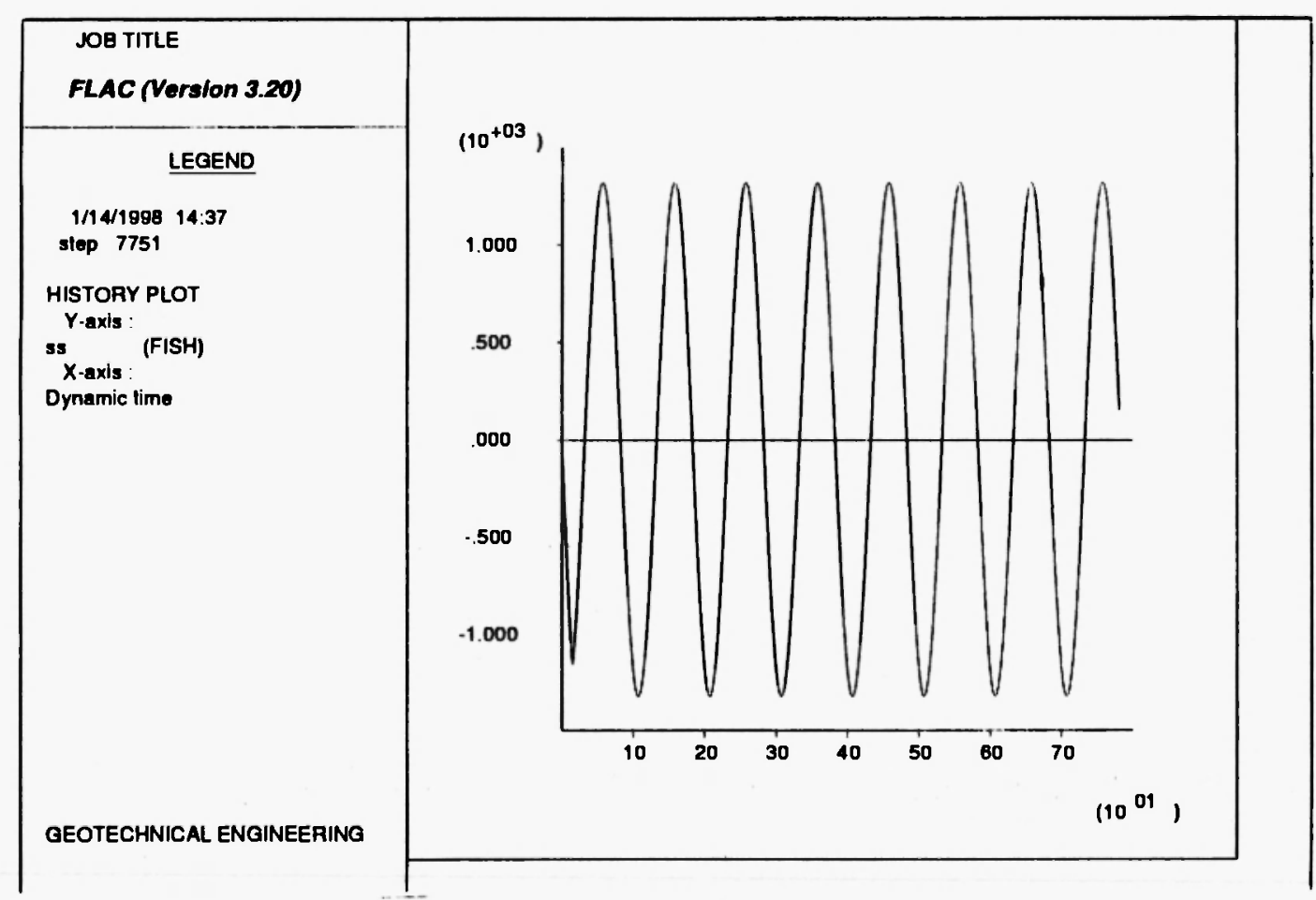

Fig. 5: The stress function at $y=0$. 
Table 1

Comparisons between the analytical and the numerical results

\begin{tabular}{|c|c|c|c|c|}
\hline Method & $\begin{array}{c}\text { Amplitude, m/sec } \\
\mathrm{f}=0.5^{*}\end{array}$ & $\begin{array}{c}\text { Amplitude, } \mathrm{m} / \mathrm{sec} \\
\mathrm{f}=1.0^{*}\end{array}$ & $\begin{array}{c}\text { Amplitude, } \mathrm{m} / \mathrm{sec} \\
\mathrm{f}=2.0^{*}\end{array}$ & $\begin{array}{c}\text { Amplitude, } \mathrm{m} / \mathrm{sec} \\
\mathrm{f}=4.0^{*}\end{array}$ \\
\hline $\begin{array}{c}\text { Analytical } \\
\text { solution }\end{array}$ & 0.973 & 0.893 & 0.593 & 0.296 \\
\hline $\begin{array}{c}\text { Numerical } \\
\text { solution }\end{array}$ & 0.965 & 0.890 & 0.603 & 0.307 \\
\hline Agreement (\%) & 0.8 & 0.3 & 1.7 & 3.6 \\
\hline
\end{tabular}

* Circular frequency

\section{CONCLUSION}

The results obtained here indicate the feasibility of representing the velocity and acceleration by means of a damper. Comparison of the analytical solution and the solution with FLAC for a longitudinal harmonic wave shows fairly close agreement which ranges from 0.8 to $3.6 \%$. It is concluded that this tool can be used in numerical modelling of the dynamic behavior of rock-structure interaction.

\section{ACKNOWLEDGEMENTS}

The research on which this paper is based is sponsored by the Center for Absorption in Science, Ministry of Immigrant Absorption, State of Israel, and by the Wolfson Family Charitable Trust Program.

\section{REFERENCES}

1. Cundall, P.A. Explicit finite difference methods in geomechanics, Proc. Numerical Methods in Engineering, EF Conference on Numerical Methods in Geomechanics, Blacksburg, Virginia, June, 1976; Vol. 1, 1976; pp. 132-150.

2. FLAC, Fast Lagrangian Analysis of Continua, Version 3.0. Vols. 1 and 2, ITASCA Consulting Group, Inc., Minneapolis, MN, 1991. 